\title{
A PROOF OF THE GENERALIZED SCHOENFLIES THEOREM
}

\author{
BY MORTON BROWN \\ Communicated by Edwin Moise, January 4, 1960
}

The following problem has been of interest for some time: Suppose $h$ is a homeomorphic embedding of $S^{n-1} \times[01]$ in $S^{n}$. Are the closures of the complementary domains of $h\left(S^{n-1} \times 1 / 2\right)$ topological $n$-cells? Recently, Barry Mazur [1] proved that the answer is affirmative if the embedding $h$ satisfies a simple "niceness" condition. In this paper we prove that the answer is affirmative with no extra conditions on $h$ required.

Definitions ANd Notation.

(1) If $Q$ is an $n$-cell then $\dot{Q}$ and $Q$ respectively denote the boundary and interior of $Q$.

(2) $I$ denotes the unit interval [01].

(3) If $f: X \rightarrow Y$ is a map, then an inverse set (under $f$ ) is a set $M \subset X$ containing at least two points, and such that for some point $y$ of $f(X), M=f^{-1}(y)$.

(4) A set $M$ is cellular in an $n$-dimensional compact metric space $S$ if there exist $n$-cells $Q_{1}, Q_{2}, \ldots$ in $S$ such that $Q_{i+1} \subset \grave{Q}_{i}$, and $\cap_{1}^{\infty} Q_{i}=M$.

Theorem 0. Let $Q$ be an $n$-cell and let $f$ map $Q$ into the $n$-sphere $S^{n}$. Suppose also that $f$ has only a finite number of inverse sets, and that these inverse sets are all in $\dot{Q}$. Then $f(Q)$ is the union of $f(\dot{Q})$ and one of its complementary domains.

Proof. Let $h=f \mid \dot{Q}$. If $f(Q) \subset f(\dot{Q})$ then $h^{-1} f$ maps $Q$ into $\dot{Q}$ and is fixed on $\dot{Q}$. This is impossible, hence $f(Q)$ intersects one of the complementary domains, say $D$, of $f(\dot{Q})$. Now $\dot{Q}$ does not separate $Q$, and $f(\dot{Q})$ separates $S^{n}$; hence $f(Q) \subset \bar{D}$. If $f(Q)$ does not contain $\bar{D}$ then $f(Q)$ has infinitely many boundary points in $D$. But by Brouwer's theorem on the invariance of domain, and the hypothesis, only a finite number of points of $f(Q) \cap D$ are boundary points of $f(Q)$. Hence $f(Q)=\bar{D}$.

Theorem 1. Let $Q$ be an $n$-cell. Suppose $M$ is a cellular subset of $\dot{Q}$.

${ }^{1}$ Actually the theorem is true if we remove the finiteness condition. Then one could prove the theorem by methods such as those in Hurewicz and Wallman's book (page 97). 
Then there is a map $f$ of $Q$ onto itself such that $f$ is fixed on $\dot{Q}$ and $M$ is the only inverse set under $f$.

Proof. Let $Q_{i}$ be a sequence of $n$-cells in $Q$ whose intersection is $M$ and such that $Q_{i+1} \subset \dot{Q}_{i}$. Let $h_{1}$ be a homeomorphism of $Q$ onto itself which is fixed on $\dot{Q}$ and such that the diameter of $h_{1}\left(Q_{1}\right)$ is less than 1. Inductively, let $h_{i+1}$ be a homeomorphism of $Q$ upon itself such that $h_{i+1}=h_{i}$ on $Q-Q_{i}$ and such that the diameter of $h_{i+1}\left(Q_{i+1}\right)$ is less than $1 /(i+1)$. Since $\left\|h_{i}-h_{i+1}\right\|$ is less than $1 /(i+1), f=\lim _{i} h_{i}$ is a map of $Q$ upon itself. The $h_{i}$ are all fixed on $\dot{Q}$, hence so is $f$. If $x, y$ are two different points of $Q$ not both in $M$, then one of them, say $x$, does not lie in some cell $Q_{i}$. Then $f(x)=h_{i}(x)$. If $y$ is in $M$ then $f(y)$ is in $h_{i}\left(\grave{Q}_{i}\right)$. But $h_{i}(x)$ does not lie in $h_{i}\left(Q_{i}\right)$. Hence if $y$ is in $M$ then $f(x) \neq f(y)$. If $y$ is not in $M$ then for some $j$, neither $x$ nor $y$ lies in $Q_{j}$. But then $f(x)=h_{j}(x)$, and $f(y)=h_{j}(y)$. But $h_{j}$ is a homeomorphism, so $f(x) \neq f(y)$. Finally, it is clear from the construction of $f$ that $f(M)$ is a point. Hence $M$ is the only inverse set under $f$.

Theorem 2. Let $S$ be a topological $n-1$ sphere in $S^{n}$ and let $D$ be one of its complementary domains. Suppose $f$ maps $\bar{D}$ onto an $n$-cell $R$ such that the only inverse set of $f$ is a cellular subset $M$ of $D$. Then $\bar{D}$ is an $n$-cell.

Proof. Let $Q$ be an $n$-cell in $D$ such that $M \subset Q$. Then $M$ is cellular in $Q$. Hence by Theorem 1 there is a map $g$ of $Q$ onto itself such that $g$ is fixed on $\dot{Q}$ and the only inverse set under $g$ is $M$. Let $g^{\prime}$ be the map of $\bar{D}$ onto itself which is the identity on $\bar{D}-Q$ and $g$ on $Q$. Then the map $f g^{-1}$ of $\bar{D}$ onto $R$ is a homeomorphism. Hence $\bar{D}$ is an $n$-cell.

Theorem 3. Let $Q$ be an n-cell and suppose $f$ maps $Q$ into $S^{n}$. Suppose also that $M \subset Q$ is the only inverse set under $f$. Then $M$ is cellular in $Q$.

Proof. It follows from Theorem 0 that $f(Q)=f(\dot{Q}) \cup D$ where $D$ is a complementary domain of $f(\dot{Q})$. Let $U$ be an open subset of $\dot{Q}$ containing $M$. Then $f(U)$ is open in $D$ and contains $f(M)$. Let $h$ be a homeomorphism of $S^{n}$ upon itself which carries $\bar{D}$ into $f(U)$ and which is fixed on some (small) neighborhood $V$ of the point $f(M)$. Let $g$ be the map of $Q$ into itself defined by:

$$
g(x)= \begin{cases}x, & x \in M, \\ f^{-1} h f(x), & x \in M .\end{cases}
$$

Since $f^{-1} h f=1$ on $f^{-1}(V), g$ is a well defined homeomorphism. Hence $g(Q)$ is an $n$-cell in $U$ containing $M$ in its interior. 
THEOREM 4. Let $f$ map $S^{n}$ onto itself such that $f$ has precisely two inverse sets $A$ and $B$. Then both $A$ and $B$ are cellular in $S^{n}$.

Proof. Let $d$ be an $n-1$ sphere in $S^{n}-(A \cup B)$ each of whose complementary domains has an $n$-cell for its closure. Let $Q$ be the $n$-cell whose boundary is $d$ and which contains $A \cup B$ in its interior. (If $d$ separates $A$ from $B$ then the theorem follows immediately from Theorem 3.) Let $f(A)=a, f(B)=b$. It follows from Theorem 0 that $f(Q)=f(\dot{Q}) \cup D$, where $D$ is that complementary domain of $f(\dot{Q})$ which contains $a \cup b$. Let $U$ be an open subset of $D$ which contains $a$ but not $b$. Then there is a homeomorphism $h$ of $S^{n}$ onto itself which carries $f(Q)$ into $U$ and which is fixed on a (small) neighborhood $V$ of $a$. Consider the map $g$ of $Q$ into itself defined by:

$$
g(x)=\left\{\begin{array}{lr}
x, & x \in A \\
f^{-1} h f(x), & x \in Q-A .
\end{array}\right.
$$

Since $f^{-1} h f=1$ on $f^{-1}(V), g$ is a well defined map. Now by inspection of $g$ one sees that the only inverse set of $g$ is $B$. Hence by Theorem 2, $B$ is cellular. In a similar manner one proves that $A$ is cellular.

THEOREM 5. Let h be a homeomorphic embedding of $S^{n-1} \times I$ into $S^{n}$. Then the closure of either complementary domain of $h\left(S^{n-1} \times 1 / 2\right)$ is an n-cell.

Proof. Let $A$ be the closure of the complementary domain of $h\left(S^{n-1} \times 1\right)$ which does not contain $h\left(S^{n-1} \times 0\right)$. Let $B$ be the closure of the complementary domain of $h\left(S^{n-1} \times 0\right)$ which does not contain $h\left(S^{n-1} \times 1\right)$. Let $f$ be a map of $S^{n}$ onto itself which carries $A, B$ respectively onto the north and south poles, which carries $h\left(S^{n-1} \times 1 / 2\right)$ onto the equator, and which has only $A$ and $B$ as inverse sets. Let $D_{A}, D_{B}$ be the complementary domains of $h\left(S^{n-1} \times 1 / 2\right)$ which contain $A, B$ respectively. By Theorem $4 A$ and $B$ are cellular. Hence $A, B$ are cellular in $D_{A}, D_{B}$ respectively. Hence, by Theorem 2, $\bar{D}_{A}$ and $\bar{D}_{B}$ are $n$-cells.

\section{REFERENCE}

1. Barry Mazur, On embeddings of spheres, Bull. Amer. Math. Soc. vol. 65 (1959) pp. 59-65.

UNIVERSITY OF MiCHIGAN 